\title{
Interplay between Process Zone and Material Heterogeneities for Dynamic Cracks
}

\author{
Fabian Barras, ${ }^{1, *}$ Philippe H. Geubelle, ${ }^{2}$ and Jean-François Molinari ${ }^{1}$ \\ ${ }^{1}$ Civil Engineering Institute, Institute of Materials Science and Engineering, \\ École Polytechnique Fédérale de Lausanne (EPFL), Station 18, 1015 Lausanne, Switzerland \\ ${ }^{2}$ Department of Aerospace Engineering, University of Illinois at Urbana-Champaign, 306 Talbot Laboratory, \\ 104 South Wright Street, Urbana, Illinois 61801, USA
}

(Received 21 April 2017; published 2 October 2017)

\begin{abstract}
Using an elastodynamic boundary integral formulation coupled with a cohesive model, we study the problem of a dynamic rupture front propagating along an heterogeneous plane. We show that small-scale heterogeneities facilitate the supershear transition of a mode-II crack. The elastic pulses radiated during front accelerations explain how microscopic variations of fracture toughness change the macroscopic rupture dynamics. Perturbations of dynamic fronts are then systematically studied with different microstructures and loading conditions. The process zone size is the intrinsic length scale controlling heterogeneous dynamic rupture. The ratio of this length scale to asperity size is proposed as an indicator to transition from quasihomogeneous to heterogeneous fracture. Moreover, we discuss how the shortening of the process zone size with increasing crack speed brings the front to interact with smaller details of the microstructure. This study shines new light on recent experiments reporting perturbations of dynamic rupture fronts, which intensify with crack propagation speed.
\end{abstract}

DOI: 10.1103/PhysRevLett.119.144101

Introduction.-Our modern understanding of fracture arose from Griffith [1] and Irwin [2] which viewed crack propagation as a thermodynamic process where, at equilibrium, the energetic cost of creating new surfaces in the material is balanced by the release of strain energy subsequent to crack advance. This theoretical framework known as linear elastic fracture mechanics (LEFM) has been successfully used over the last 50 years to predict the stability of flaws in engineering materials. Consequently LEFM was extended to cracked bodies far from equilibrium, i.e., to dynamic fracture mechanics [3,4]. Experiments on brittle solids showed that this dynamic theory of fracture gives good prediction for slow crack propagation but is unsuitable to describe fast rupture events where the crack front speed is a significant fraction of material shear wave speed $c_{s}$. In particular, linear elastic theory overestimates the propagation speed and significantly underestimates the dissipated energy. For a review of dynamic fracture experiments, the reader is referred to [5-9]. A three-stage transition is universally observed within brittle materials, usually referred to as "mirror," "mist," and "hackle" in reference to the postmortem appearance of fracture surface. At low rupture velocity, fracture surfaces are planar and smooth (mirror) and crack dynamics is thereby well predicted by LEFM theory. As crack speed increases, the rupture remains in plane but fracture surface roughens (mist), followed by a stage characterized by the formation of out-of-plane microbranches (hackle), and finally the onset of macroscopic branching. This transition observed in various brittle materials $[7,10]$ and at different scales [11,12] explains how linear elasticity fails at describing fast rupture events where the front starts to interplay with the microstructure and/or dynamic instabilities and becomes a heterogeneous fracture problem [13-16]. An extension of LEFM to heterogeneous problems was proposed by Rice [17] and Gao and Rice [18] who gave a firstorder estimation of the stress intensity perturbation caused by crack front distortion in presence of tougher asperities. Recently, Ponson [19] reviewed how this approach can successfully predict the roughness of slow rupture front in brittle disordered material as long as crack front can be viewed as a unique elastic line. The complex mechanisms driving fast crack propagation occur however at a smaller scale where fracture develops along a finite length. In this context, numerical models have a great potential to bring new insight on the interaction of a dynamic front with material heterogeneities. However, the small spatiotemporal scales characterizing this process require a very fine discretization of the fracture plane and explain why dynamic heterogeneous fracture remains overlooked.

In this manuscript, we investigate the interaction of a dynamic rupture front with small-scale heterogeneities. The objective is to understand how rupture dynamics is perturbed when the average fracture properties are identical but their statistical distribution changes. The rupture is assumed to propagate along a weak interface under mode-II plane strain conditions. This heterogeneous fracture problem is solved using a boundary integral formulation of the elastodynamics equation proposed by Breitenfeld and Geubelle [20]. This approach, initially developed by Geubelle and Rice [21], allows an extremely fine discretization of the fracture plane associated with a prescribed cohesive model, particularly suited to describe phenomena occurring in the immediate vicinity of dynamic crack tips [22]. 
Problem description: Geometry and elastodynamics.Let us consider two semi-infinite linearly elastic solids under plane strain conditions with the top $(y>0)$ and bottom $(y<0)$ displacements fields respectively denoted by $\boldsymbol{u}^{+}(x, y, t)$ and $\boldsymbol{u}^{-}(x, y, t)$. Along the interface $(y=0)$, the two half spaces are initially bounded in static equilibrium under a uniform in-plane shear (mode-II) loading $\tau_{0}$. The initial conditions can then be summarized as

$$
\begin{aligned}
\dot{\boldsymbol{u}}^{+}(x, y, t=0) & =\dot{\boldsymbol{u}}^{-}(x, y, t=0)=0, \\
\boldsymbol{\sigma}_{x y}^{+}(x, y, t=0) & =\boldsymbol{\sigma}_{x y}^{-}(x, y, t=0)=\tau_{0},
\end{aligned}
$$

with $\dot{\boldsymbol{u}}=(\partial \boldsymbol{u} / \partial t)$ and $\boldsymbol{\sigma}$ the Cauchy stress tensor. Because of the spectral nature of the numerical scheme which is based on a Fourier series representation of the interface quantities, the domain of interest is periodic with period $X$, i.e., $\boldsymbol{u}(0, y, t)=\boldsymbol{u}(X, y, t)$. At time $t=0$, a crack of length $a$ is inserted at the left corner of the domain and starts to grow dynamically in the right direction while left tip propagation is prevented. Crack propagation is studied while $a<\frac{2}{3} X$ to neglect the effect of periodic boundary conditions. Figure 1 illustrates the studied brittle fracture process, which is constrained to the interface $(y=0)$. Across the interface, the displacement discontinuity is defined as

$$
\boldsymbol{\delta}(x, t)=\boldsymbol{u}^{+}\left(x, y=0^{+}, t\right)-\boldsymbol{u}^{-}\left(x, y=0^{-}, t\right) .
$$

The interface resists crack motion with $G_{c}^{H}$, which corresponds to twice the material surface energy and is named toughness in this manuscript. Far from the initiation site $\left(x>L_{\mathrm{hom}}\right)$, the toughness presents dispersion in the idealized form of constant width $w$ stripes alternately weaker $\left(G_{c}^{\text {weak }}<G_{c}^{H}\right)$ and tougher $\left(G_{c}^{\text {strong }}>G_{c}^{H}\right)$. This arrangement of asperities is designed to keep the macroscopic fracture toughness unchanged: $\left\langle G_{c}\right\rangle=0.5\left(G_{c}^{\text {weak }}+G_{c}^{\text {strong }}\right)=G_{c}^{H}$. Plane strain conditions prevent any crack front distortions

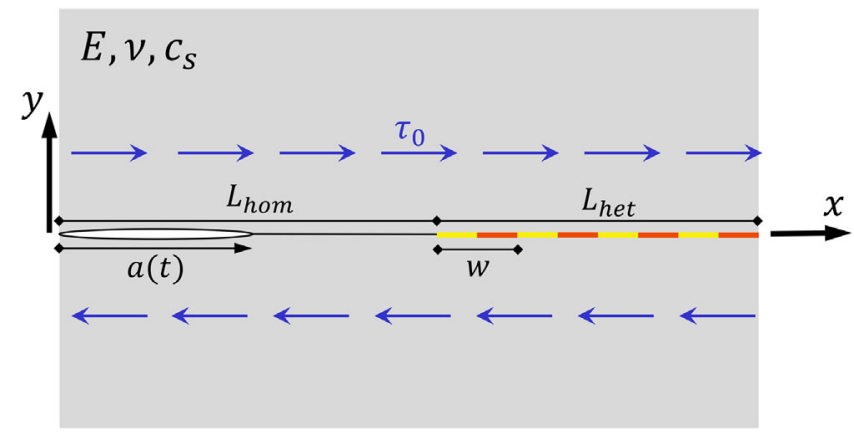

FIG. 1. Geometry of the in-plane heterogeneous fracture problem. A crack of length $a$ is inserted along an interface with constant macroscopic toughness $G_{c}^{H}$ at rest under a uniform shear loading $\tau_{0}$. The interface is made of a homogeneous portion $L_{\mathrm{hom}}$ and a region with a heterogeneous toughness $L_{\text {het }}$ in form of alternately weaker (yellow)/tougher (orange) stripes of constant width $w$. during failure and the straight crack front successively breaks this array of asperities.

Problem description: Numerical scheme.-The dynamic fracture problem is solved with the aid of the spectral scheme [20,21], a spectral form of the elastodynamic boundary integral relations between the displacements $\boldsymbol{u}^{ \pm}$along the fracture plane and the corresponding traction stress $\boldsymbol{\tau}(x, t)$. The numerical method allows for a detailed description of the evolution of the displacements, velocities, and traction stresses along the interface, especially in the failure zone captured with the aid of a cohesive failure model relating the displacement jump in the slip direction, $\delta_{x}$, and the interface strength

$$
\tau^{\mathrm{str}}(x, t)=\tau_{c}(x)\left\{1-\delta_{x}(x, t) / \delta_{c}(x)\right\} .
$$

In (3), $\tau_{c}$ and $\delta_{c}$ respectively denote the failure strength and critical slip, and $\{\xi\}=\xi$ if $\xi>0$ and 0 otherwise. The corresponding value of the toughness is $G_{c}(x)=$ $\frac{1}{2} \tau_{c}(x) \delta_{c}(x)$. The interface is typically discretized with 65536 nodes in the simulations presented hereafter. Details about the numerical method are provided to the reader as Supplemental Material [23].

Problem description: Material properties and fracture initiation.-The material and failure properties are given to the reader in [23] for the sake of reproducibility, but the conclusions drawn in the manuscript are independent from this choice and results are hereafter presented in adimensional scales. Based on the expression of the stress intensity factor $K_{I I}=\tau_{0} \sqrt{\pi(a / 2)}$ for static shear-loaded crack in an infinite medium with Young's modulus $E$ and Poisson's ratio $\nu[2,24]$, we compute the critical crack size $L_{c}$ satisfying Griffith's failure criterion:

$$
L_{c}=\frac{2 K_{I I}^{2}}{\pi \tau_{0}^{2}}=\frac{2 G_{c}^{H}}{\pi \tau_{0}^{2}} \frac{E}{\left(1-\nu^{2}\right)}=\frac{\tau_{c}^{H} \delta_{c}}{\pi \tau_{0}^{2}} \frac{E}{\left(1-\nu^{2}\right)},
$$

often referred to as Griffith crack length [25]. Rupture is initiated in this work by slowly growing an infinitesimal crack to its critical length $a \simeq L_{c}$ and dynamic rupture begins $(t=0)$ when it starts to grow spontaneously.

The effect of heterogeneous microstructure.-We start our study with the rupture of a perfectly homogeneous interface $\left(L_{\text {het }}=0\right)$ initially at rest with $\tau_{0}=0.22 \tau_{c}^{H}$. As predicted by the crack tip equation of motion [4], the constant loading makes the crack continuously accelerate up to the upper limit represented by the Rayleigh wave speed $c_{R}$ [Fig. 2(a)]. While keeping the same system on average, we increase its statistical dispersion after $L_{\text {hom }}=$ $2 L_{c}$ by introducing stripes of weaker $\left(\tau_{c}^{\text {weak }}=\frac{1}{3} \tau_{c}^{H}\right)$ and stronger $\left(\tau_{c}^{\text {strong }}=\frac{5}{3} \tau_{c}^{H}\right)$ heterogeneities of width $w=0.6 L_{c}$ according to Fig. 1. This heterogeneous microstructure leads, however, to a dramatic change in rupture dynamics, which is presented in Fig. 2(b). After a first propagation phase at a sub-Rayleigh regime $x / L_{c}<15$, the rupture 

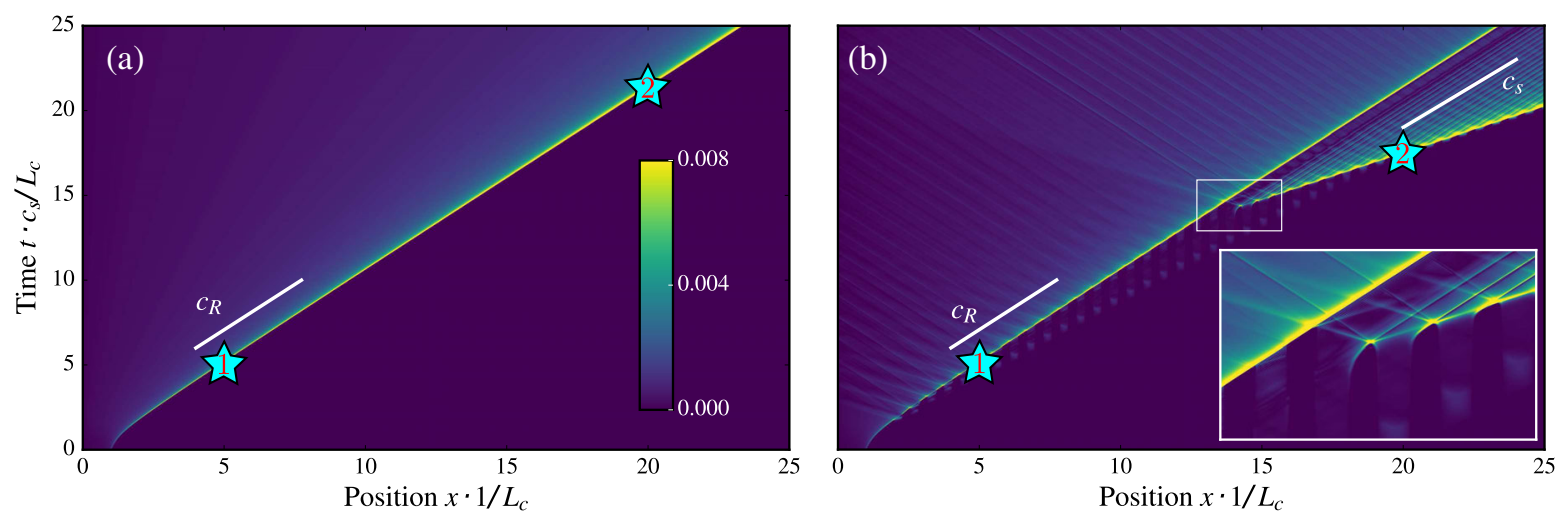

FIG. 2. Space-time diagrams of two macroscopically equivalent dynamic fracture events where the normalized slip velocity $\dot{\delta}_{x} / c_{s}$ is shown using the same color scale while the crack tip position at $a_{1}=5 L_{c}$ and $a_{2}=20 L_{c}$ is highlighted with cyan stars. In (a) the crack grows on a perfectly homogeneous interface, while in (b) the rupture front interacts with smaller-scale heterogeneities.

front is able to exceed the Rayleigh wave speed and $c_{s}$ (see [23] for additional representations of these rupture events). The supershear transition of mode-II crack is fundamental in the understanding of earthquake dynamics [26-28] and several works [25,29-33] studied how a propagating front may eventually get supershear; the time-growing pulse radiated ahead of an accelerating shear crack causes the nucleation of a secondary supershear front if its maximum amplitude exceeds interface strength. For a rupture growing from $a=L_{c}$, Andrews [25] was the first to report how this transition occurs at a specific crack size, which depends on the initial ratio between interface strength and prestress, the so-called seismic ratio $S=\left(\tau_{c}^{H}-\tau_{0}\right) / \tau_{0}$. Following the same formalism, Fig. 3 summarizes our quantitative study

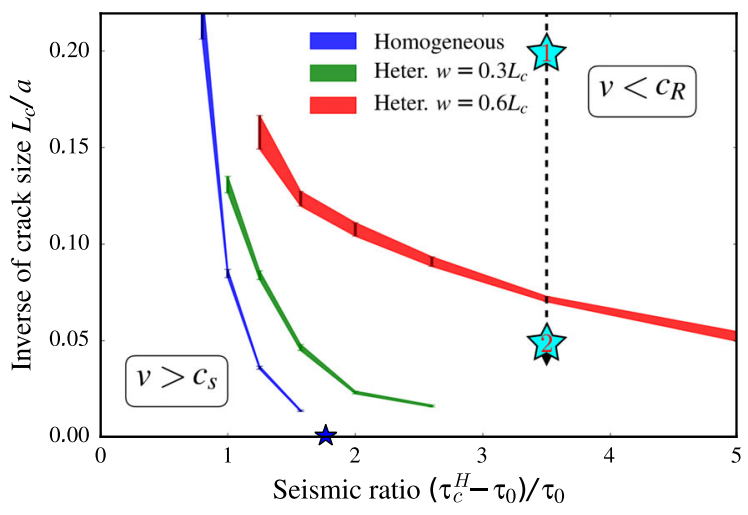

FIG. 3. Effect of heterogeneities on the supershear transition. Color curves trace the observed boundary between sub-Rayleigh and supershear regimes for different loading conditions (seismic ratio) and toughness distribution. The dashed arrow draws the trajectory of ruptures of Fig. 2 where the crack is initially in the sub-Rayleigh regime (cyan star at $a_{1}=5 L_{c}$ ) and grows toward a size (cyan star at $a_{2}=20 L_{c}$ ) where it either crosses the boundary toward supershear regime [as in the interface of Fig. 2(b) with $w=0.6 L_{c}$ ], or not [as in the homogeneous interface of Fig. 2(a)]. The dark blue star shows the maximum seismic ratio allowing supershear crack in homogeneous plane strains interface [25,34]. of this supershear criterion for different loading conditions and toughness distributions. With a plane strain homogeneous setup, we meet the transition dynamics reported in the literature $[25,34]$. The comparison with the heterogeneous setup reveals how increasing the microscopic toughness dispersion facilitates the supershear transition by both extending the limiting seismic ratio and reducing the required transition length. The explanation is found in the increase of elastic radiations caused by the heterogeneous microstructure. During homogeneous rupture, the slip velocities profile is smooth and high velocities are concentrated within the process zone. In the presence of heterogeneities, elastic waves are continuously emitted from the propagating tip resulting in a succession of pulses visible in the slip velocity profile. The inset of Fig. 2(b) presents a collective mechanism where the rupture of tougher asperities creates waves which are later helping the rupture of the neighboring asperities. Supershear transition caused by a favorable heterogeneity has been reported in several works in the context of earthquake dynamics in the presence of heterogeneous prestress or toughness along the slip plane [30,35-37] or with off-fault elastic heterogeneity [38,39]. Following this benchmark problem of dynamic fracture, our study reveals how a collective mechanism occurring at the smaller scale of an heterogeneous interface can deeply impact the macroscopic rupture dynamics. Moreover, Fig. 3 emphasizes the importance of the details of microscale properties which are systematically discussed in the next section.

Transition from homogeneous to heterogeneous fracture.-In the system considered, two parameters characterize the heterogeneity of the interface: the size $w$ of the asperities and the toughness contrast $G_{c}^{\text {strong }} / G_{c}^{\text {weak }}$. The rupture speed is another important parameter, which constantly increases in the preceding simulations because of the load-controlled setup. After an identical smooth initiation, a progressively decaying loading $\tau_{0}(a)$ allows for crack growth at a constant speed along a perfectly homogeneous interface ( $K$-controlled setup). The same loading 

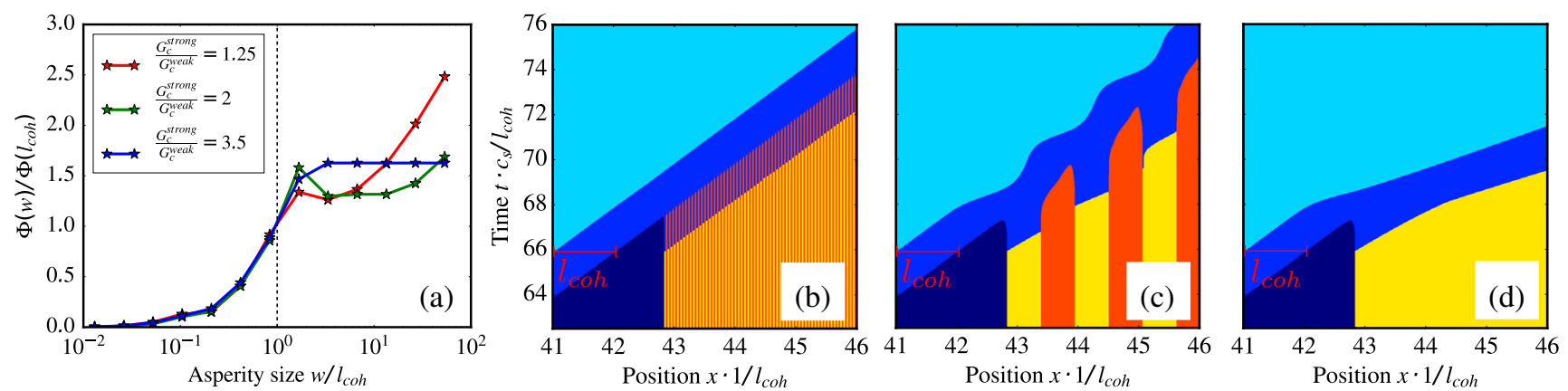

FIG. 4. The process zone size is the length scale controlling crack front interaction with heterogeneities. For $v=0.5 c_{s}$ and a toughness ratio of 3.5, colors in plots (b)-(d) divide broken surface (sky blue), cohesive zone (blue), and intact interface which is either dark blue (average properties), yellow (weaker properties), or orange (tougher properties). (a) Normalized increase of slip velocity as a function of interface heterogeneity, namely, asperity size and toughness ratio for $v=0.5 c_{s}$. (b) Asperities are much smaller than $l_{\text {coh }}$ leading to quasihomogeneous dynamics. (c) Collective interaction between depinning events when $w$ is in the range of $l_{\text {coh }}$ leading to a significant impact on rupture dynamics. (d) When the asperities are much larger than $l_{\text {coh }}$, the material is macroscopically heterogeneous.

conditions are further replicated to rupture fronts meeting an heterogeneous region far from the initiation site $\left(L_{\mathrm{hom}}=L_{\mathrm{het}}=5 L_{c}\right)$. As described in the previous section [see Fig. 2(b)], one signature of the interplay of dynamic crack front with heterogeneities is the slip pulses emitted during the successive front accelerations. We measure and compare then the maximum slip velocity $\max \left\{\dot{\delta}_{x}\right\}$ observed when the front breaks the heterogeneous region and compare it to the value measured when the interface is perfectly homogeneous $\max \left\{\dot{\delta}_{x}^{H}\right\}$. The normalized increase in slip velocity

$$
\Phi\left(w, \frac{G_{c}^{\text {strong }}}{G_{c}^{\text {weak }}}\right)=\frac{\max \left\{\dot{\delta}_{x}\right\}-\max \left\{\dot{\delta}_{x}^{H}\right\}}{\max \left\{\dot{\delta}_{x}^{H}\right\}}
$$

is then used as an indicator of crack front perturbation for a given heterogeneous microstructure. A progressive increase of slip velocities is observed when increasing the toughness ratio between weaker and stronger asperities. However, when changing the size of the asperities, the evolution of $\Phi$ is not monotonic and depends on a critical asperity size. The width of the process zone, $l_{\text {coh }}$, namely the distance over which the interface evolves from intact to broken, is the characteristic length scale emerging from this $K$-controlled setup. Figure 4(a) presents how the increase in slip velocity measured with different asperity sizes and toughness ratios collapses after being normalized by the size of the process zone (data before normalization are available in [23]). Three characteristic behaviors emerge from this normalization. When $w \sim l_{\text {coh }}$ [Fig. 4(c)], the heterogeneous interface develops a collective mechanism similar to the one discussed in Fig. 2(b) and leads to a significant perturbation of the rupture dynamics. The effect of heterogeneities quickly decays as $w<l_{\text {coh. }}$. When several weaker and stronger heterogeneities are contained within the process zone [Fig. 4(b)], their fracture properties are averaged and homogenized resulting in a rupture dynamics identical to the perfectly homogeneous setup. Finally when $w \gg l_{\text {coh }}$, the collective interaction between asperities ceases as the elastic waves have time to dissipate within the bulk between two depinning events. In this macroscopically heterogeneous interface, $l_{\text {coh }}$ stops characterizing the rupture dynamics which meets the predictions of the singular fracture theory where the crack speed instantaneously adapts to a change in fracture toughness [Fig. 4(d)]. These characteristic behaviors controlled by the process zone size are universally observed with different types of heterogeneity $G_{c}^{\text {strong }} / G_{c}^{\text {weak }}[$ Fig. 4(a)] as well as different crack front speeds (inset of Fig. 5).

Process zone size in dynamic fracture.-At the very vicinity of crack tip, one should admit a region, the process

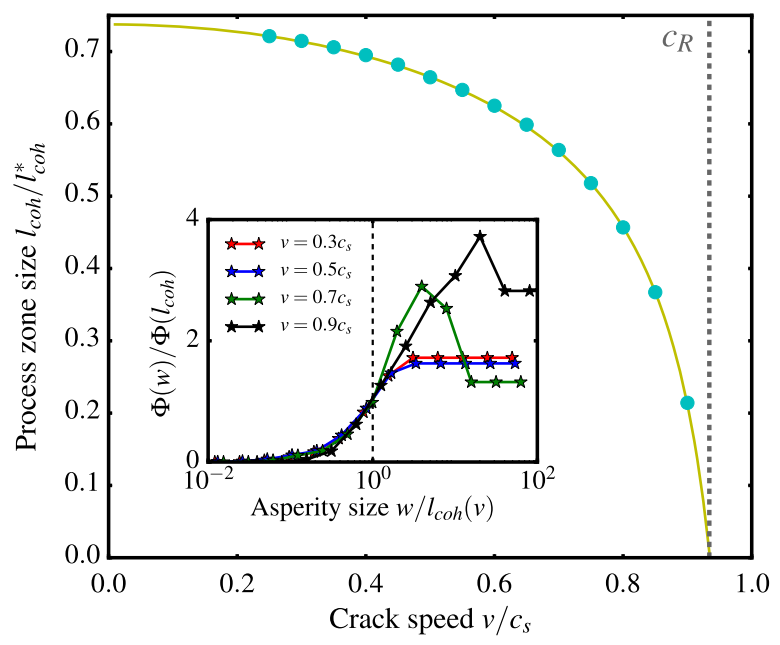

FIG. 5. Faster crack fronts interact with smaller heterogeneities. The main plot details how the process zone size contracts as the crack accelerates toward $c_{R}$. Cyan dots show simulation data compared with the theoretical prediction in yellow (details are provided in [23]). The inset shows the increase of slip velocity as function of asperity size for $G_{c}^{\text {strong }} / G_{c}^{\text {weak }}=3.5$ and different crack speeds using $l_{\text {coh }}(v)$ for normalization to collapse data. 
zone, where nonlinear dissipative processes are regularizing the square root singularity. The nature of these nonlinear processes is far from being understood and should certainly be highly dependent on the material. Cohesive models arose from Dugdale [40] and Barenblatt [41] approaches which propose an elegant way of modeling these processes without losing the universality of LEFM theory. In this nonsingular framework, $l_{\text {coh }}$ scales as

$$
\left(\frac{K_{I I}}{\tau_{c}^{H}}\right)^{2} \sim l_{\mathrm{coh}}^{*}=\frac{G_{c}^{H}}{\left(\tau_{c}^{H}\right)^{2}} \frac{E}{\left(1-\nu^{2}\right)}=\frac{1}{2} \frac{\delta_{c}}{\tau_{c}^{H}} \frac{E}{\left(1-\nu^{2}\right)},
$$

for a crack at equilibrium with the linear slip-weakening law of Eq. (3). Within the $K$-controlled setup, the process zone has then a constant size along the homogeneous portion of the interface as highlighted in Figs. 4(b)-4(d). We thereby measured its length at different rupture speeds in Fig. 5 and observed a shrinkage of $l_{\text {coh }}$ as $v$ increased. This relativistic process zone contraction as $v$ approaches the information speed is indeed predicted theoretically [42-44] and was recently measured in experiments [45]. $l_{\text {coh }}$ is even expected to be infinitely small when $v$ approaches $c_{R}$. The fine level of discretization provided by the boundary element model enables us to capture this process zone contraction. Since $l_{\text {coh }}$ is the characteristic length governing the interaction of crack front with material heterogeneities, its contraction implies that faster cracks are perturbed by smaller heterogeneities or defects along their path.

Discussion.-Taking advantage of the fine discretization allowed by the boundary integral formulation [20], we investigate numerically the interplay of a dynamic crack front with heterogeneities. A planar straight crack under plane strain conditions interplays with an idealized microstructure made of equispaced stripes of weaker and stronger areas. We reveal a complex mechanism where the nucleation and coalescence of crack fronts within the heterogeneous microstructure radiates elastic waves helping the rupture of neighboring asperities. This collective process occurring at the scale of the heterogeneous microstructure directly changes the macroscopic dynamics and facilitates the supershear transition. We present then how the size of the process zone is the length scale characterizing the perturbation of a dynamic front by material heterogeneities. This observation was confirmed with different subRayleigh front speeds and heterogeneous microstructures. Moreover, the process zone size decreases with the crack velocity, shrinking to zero as $v$ approaches $c_{R}$. As the rupture front accelerates toward $c_{R}$, it interacts therefore with smaller material heterogeneities (asperities, defects). We suggest that this process zone contraction amplifies thereby the dynamic instabilities and roughens the fracture surface of an accelerating crack front. This study shines a new light on the interplay between a dynamic rupture front and the material-heterogeneity length scales. The observations and conclusions drawn in this manuscript have direct implications in the understanding of earthquake dynamics [26,28,31] (supershear rupture) as well as the evolution observed in the fracture behavior of materials with increasing rupture speed $[10,13,16]$ (interaction of crack with defects or microstructures, dynamic instabilities).

This work was supported by the Swiss National Science Foundation (Grant No. 162569).

*Corresponding author. fabian.barras@epfl.ch

[1] A. A. Griffith, Phil. Trans. R. Soc. A 221, 163 (1921).

[2] G. Irwin, J. Appl. Mech. 24, 361 (1957).

[3] B. V. Kostrov and S. Das, Principles of Earthquake Source Mechanics, Cambridge Monographs on Mechanics and Applied Mathematics (Cambridge University Press, Cambridge, England, 1988).

[4] L. B. Freund, Dynamic Fracture Mechanics, Cambridge Monographs on Mechanics and Applied Mathematics (Cambridge University Press, Cambridge, England, 1990).

[5] K. Ravi-Chandar and W. G. Knauss, Int. J. Fract. 25, 247 (1984).

[6] K. Ravi-Chandar and W. G. Knauss, Int. J. Fract. 26, 65 (1984).

[7] K. Ravi-Chandar and W. G. Knauss, Int. J. Fract. 26, 141 (1984).

[8] J. Fineberg and M. Marder, Phys. Rep. 313, 1 (1999).

[9] E. Bouchbinder, T. Goldman, and J. Fineberg, Rep. Prog. Phys. 77, 046501 (2014).

[10] J. Scheibert, C. Guerra, F. Clari, D. Dalmas, and D. Bonamy, Phys. Rev. Lett. 104 (2010).

[11] J. A. Hauch, D. Holland, M. P. Marder, and H. L. Swinney, Phys. Rev. Lett. 82, 3823 (1999).

[12] T. Cramer, A. Wanner, and P. Gumbsch, Phys. Rev. Lett. 85, 788 (2000).

[13] E. Sharon, S. P. Gross, and J. Fineberg, Phys. Rev. Lett. 76, 2117 (1996).

[14] K. Ravi-Chandar and B. Yang, J. Mech. Phys. Solids 45, 535 (1997).

[15] A. Rabinovitch, G. Belizovsky, and D. Bahat, Phys. Rev. B 61, 14968 (2000).

[16] C. Guerra, J. Scheibert, D. Bonamy, and D. Dalmas, Proc. Natl. Acad. Sci. U.S.A. 109, 390 (2012).

[17] J. R. Rice, J. Appl. Mech. 52, 571 (1985).

[18] H. Gao and J. R. Rice, J. Appl. Mech. 56, 828 (1989).

[19] L. Ponson, Int. J. Fract. 201, 11 (2016).

[20] M. S. Breitenfeld and P. H. Geubelle, Int. J. Fract. 93, 13 (1998).

[21] P. H. Geubelle and J. R. Rice, J. Mech. Phys. Solids 43, 1791 (1995).

[22] F. Barras, D. S. Kammer, P. H. Geubelle, and J.-F. Molinari, Int. J. Fract. 189, 149 (2014).

[23] See Supplemental Material at http://link.aps.org/ supplemental/10.1103/PhysRevLett.119.144101 for additional information regarding the numerical method and some of the results discussed in the manuscript.

[24] T. L. Anderson, Fracture Mechanics: Fundamentals and Applications, 3rd ed. (Taylor \& Francis, Boca Raton, FL, 2005). 
[25] D. J. Andrews, J. Geophys. Res. 81, 5679 (1976).

[26] R. J. Archuleta, J. Geophys. Res. 89, 4559 (1984).

[27] A. J. Rosakis, O. Samudrala, and D. Coker, Science 284, 1337 (1999).

[28] M. Mello, H. S. Bhat, and A. J. Rosakis, J. Mech. Phys. Solids 93, 153 (2016).

[29] R. Burridge, Geophys. J. Int. 35, 439 (1973).

[30] E. M. Dunham, P. Favreau, and J. M. Carlson, Science 299, 1557 (2003).

[31] F. X. Passelegue, A. Schubnel, S. Nielsen, H. S. Bhat, and R. Madariaga, Science 340, 1208 (2013).

[32] S. G. Psakhie, E. V. Shilko, M. V. Popov, and V. L. Popov, Phys. Rev. E 91 (2015).

[33] I. Svetlizky, D. Pino Munoz, M. Radiguet, D. S. Kammer, J.-F. Molinari, and J. Fineberg, Proc. Natl. Acad. Sci. U.S.A. 113, 542 (2016).

[34] E. M. Dunham, J. Geophys. Res. 112, B07302 (2007).

[35] Y. Liu and N. Lapusta, J. Mech. Phys. Solids 56, 25 (2008).
[36] E. Fukuyama and K. B. Olsen, Pure Appl. Geophys. 159, 2047 (2002).

[37] D. Kammer, D. Pino Munoz, and J. Molinari, J. Mech. Phys. Solids 88, 23 (2016).

[38] X. Ma and A. Elbanna, Geophys. J. Int. 203, 664 (2015).

[39] G. Albertini and D. S. Kammer, J. Geophys. Res.: Solid Earth 122, 6625 (2017).

[40] D. Dugdale, J. Mech. Phys. Solids 8, 100 (1960).

[41] G. Barenblatt, Adv. Appl. Mech. 7, 55 (1962).

[42] J. R. Rice, in Physics of the Earth's Interior (Italian Physical Society and North Holland, New-York, 1980) pp. 555-649.

[43] J. W. Morrissey and J. R. Rice, J. Mech. Phys. Solids 46, 467 (1998).

[44] D. V. Kubair, P. H. Geubelle, and Y. Y. Huang, J. Mech. Phys. Solids 50, 1547 (2002).

[45] I. Svetlizky and J. Fineberg, Nature (London) 509, 205 (2014). 